\title{
Outcomes of second-tier rapid response activations in a tertiary referral hospital: A prospective observational study
}

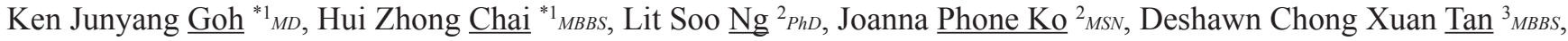
Hui Li Tan ${ }^{2} M S N$, Constance Wei-Shan Teo ${ }^{4}{ }_{R R T}$, Ghee Chee Phua ${ }^{1}{ }_{F C C P}$, Qiao Li Tan ${ }^{1} M B B S$

\begin{abstract}
Introduction: A second-tier rapid response team (RRT) is activated for patients who do not respond to first-tier measures. The premise of a tiered response is that first-tier responses by a ward team may identify and correct early states of deterioration or establish goals of care, thereby reducing unnecessary escalation of care to the RRT. Currently, utilisation and outcomes of tiered RRTs remain poorly described.
\end{abstract}

Methods: A prospective observational study of adult patients (age $\geq 18$ years) who required RRT activations was conducted from February 2018 to December 2019.

Results: There were 951 consecutive RRT activations from 869 patients and $76.0 \%$ patients had a National Early Warning Score (NEWS) $\geq 5$ at the time of RRT activation. The majority (79.8\%) of patients required RRT interventions that included endotracheal intubation (12.7\%), point-of-care ultrasound (17.0\%), discussing goals of care (14.7\%) and intensive care unit (ICU) admission (24.2\%). Approximately 1 in $3(36.6 \%)$ patients died during hospitalisation or within 30 days of RRT activation. In multivariate analysis, age $\geq 65$ years, NEWS $\geq 7$, ICU admission, longer hospitalisation days at RRT activation, Eastern Cooperative Oncology Group performance scores $\geq 3$ (OR [odds ratio] 2.24, 95\% CI [confidence interval] 1.45-3.46), metastatic cancer (OR 2.64, 95\% CI 1.71-4.08) and haematological cancer (OR 2.78, 95\% CI 1.84-4.19) were independently associated with mortality.

Conclusion: Critical care interventions and escalation of care are common with second-tier RRTs. This supports the need for dedicated teams with specialised critical care services. Poor functional status, metastatic and haematological cancer are significantly associated with mortality, independent of age, NEWS and ICU admission. These factors should be considered during triage and goals of care discussion.

Ann Acad Med Singap 2021;50:838-47

Keywords: Clinical deterioration, critical care, intensive care, mortality, rapid response system, rapid response team

\section{INTRODUCTION}

Despite its widespread adoption, rapid response systems (RRS) and rapid response teams (RRT) vary significantly in composition and set-up..$^{1-3}$ While implementation of RRSs appear to be associated with reduced cardiac arrest rate and improved mortality, ${ }^{4,5}$ their optimal composition, activation criteria and how they should be evaluated, remain controversial. ${ }^{6-9}$ It is likely that RRS staffing, calling criteria and clinical pathways are influenced by the available expertise, patient case-mix and resources in each hospital.
Singapore General Hospital is a 1,800-bed tertiary referral hospital that encompasses a national cancer centre, specialist haematology service, and a 550-bed community hospital. Being a large tertiary centre with highly specialised clinical services, our RRS has evolved to include specialised (medical and surgical) and dedicated (separate from our cardiac arrest team) RRTs. Our RRS is based on a multitiered response. The primary or ward team leads the first-tier response (where initial clinical deterioration triggers a clinical review by the ward team) and makes the decision for RRT activation

\footnotetext{
${ }^{1}$ Department of Respiratory and Critical Care Medicine, Singapore General Hospital, Singapore

${ }^{2}$ Speciality Nursing, Nursing Division, Singapore General Hospital, Singapore

${ }^{3}$ Yong Loo Lin School of Medicine, National University of Singapore, Singapore

${ }^{4}$ Respiratory Therapy Unit, Singapore General Hospital, Singapore

Correspondence: Dr Ken Junyang Goh, Department of Respiratory and Critical Care Medicine, Singapore General Hospital, Academia, 20 College Road, Singapore 169856 .

Email: ken.goh.junyang@singhealth.com.sg

* Joint first authors
} 


\section{CLINICAL IMPACT}

\section{What is New}

- Many patients with rapid response team (RRT) activations require 1 or more RRT interventions. Clinical factors such as poor functional status and metastatic and/or haematological cancer were significantly associated with poorer survival.

\section{Clinical Implications}

- Second-tier RRTs should be dedicated teams capable of providing specialised critical care services. - Institutions should continually address potential barriers to RRT activation and consider measures to minimise delayed activations, such as the use of early warning scores. Clinical factors associated with poor outcomes should be taken into consideration during triage and goals of care discussion.

(second-tier response). The premise of a tiered response is that a first-tier response by the ward team allows early states of deterioration to be identified and corrected, and goals of care to be established, thereby reducing unnecessary escalation of care to the RRT. A tiered RRS response is not a new concept and it may promote more efficient use of critical care resources with improved staff satisfaction. ${ }^{10,11}$ Introduction of a tiered response has been reported to be associated with lower intensive care unit (ICU) mortality for patients admitted after RRT activations. ${ }^{12}$ This plausibly relates to a decision by the ward team not to escalate the level of care, or establishing limits on the extent of medical therapy, for patients who are unlikely to benefit from aggressive medical therapy. ${ }^{12}$ Tiered responses are commonplace in pre-hospital emergency services and increasingly being used in trauma centres to facilitate efficient use of resources. ${ }^{13-15}$

There is however, limited evidence describing the utilisation of a multitiered RRS in terms of RRT interventions, patient outcomes and prognostic markers. These factors are important for planning RRT composition, critical care resource management, and guiding RRT triaging and escalation of care. We aimed to evaluate second-tier RRT interventions and patient outcomes in a tertiary referral hospital. Additionally, we sought to investigate factors associated with mortality in patients requiring a second-tier RRT response.

\section{METHODS}

We conducted a prospective observational study of RRT activations from February 2018 to December 2019.
Prior to the implementation of RRTs, resuscitation at the ward level was performed by the primary or ward team. Ward teams were informed of deteriorating patients based on nurses' concerns, or critical values for single parameter vital signs such as systolic blood pressure $\leq 90 \mathrm{mmHg}$. RRTs were implemented in our institution in February 2018.

The medical RRT is also known as the Medical ICU Acute Response Team (SMART). The SMART team comprises an intensivist consultant (or registrar) from the medical ICU, medical junior resident (when available), critical care advanced practice nurse and a respiratory therapist. The medical emergency response is 3-tiered, where clinical deterioration or concerns from nursing staff is first escalated to the ward team (first tier), followed by activation of the RRT (second tier) if the patient requires escalation to a higher care ward or does not respond to first-tier management. The cardiac arrest team is the third tier.

No early warning system existed in our hospital at the time of our study. Similar to pre-RRT implementation, activation of the first tier (ward team) was based on deterioration of single parameter vital signs or concerns of ward and nursing staff. Ward teams were encouraged to activate the RRT if they were concerned about the patient or escalation of care is likely to be required, in the presence of any of the following criteria: respiratory rate $\leq 8$ or $\geq 25$ breaths per minute; $\mathrm{SpO}_{2}$ $\leq 91 \%$ on supplemental oxygen; systolic blood pressure $\leq 90$ or $\geq 220 \mathrm{mmHg}$; heart rate $\leq 40$ or $\geq 131$ beats per minute; and acute change in Glasgow Coma Scale or unarousable patient. The 24-hour medical RRT service is supported by a night team of a medical registrar and respiratory therapist. Since its inception, there have been an average of 88 medical activations per month, with a utilisation rate (RRT dose) of approximately 22 activations per 1,000 medical admissions.

We recruited adult patients (age $\geq 18$ years) by medical disciplines who required RRT activations during daytime hours ( $8 \mathrm{am}-5 \mathrm{pm})$. Patient demographics, clinical characteristics and RRT interventions were recorded prospectively. National Early Warning Scores (NEWS) ${ }^{16}$ at the time of RRT activation were determined. Comorbidities as defined by Charlson Comorbidity Index $(\mathrm{CCI})^{17}$ and Eastern Cooperative Oncology Group (ECOG) performance status ${ }^{18}$ scores based on functional status prior to hospitalisation were recorded. Patients with active malignancies or patients treated for malignancy in the last 5 years were considered to have an underlying malignancy as a comorbidity. Lymphoma, leukaemia and multiple myeloma were defined as haematological malignancies. 
Patients who had more than 1 RRT activation during the same hospitalisation period were considered to have a repeat RRT activation. The reason for RRT activation was also categorised into airway concerns (included decreased airway patency, inability to protect airway, drowsiness or loss of consciousness); respiratory failure (included respiratory distress or tachypnoea); cardiovascular concerns (included hypotension, significant hypertension, tachycardia, bradycardia or arrhythmias); metabolic derangements; and primary team concern or request for closer monitoring. RRT response times were determined based on the time interval between receiving the call activation and time of arrival of the RRT by the bedside.

RRT interventions were defined as emergent bedside interventions performed in the general ward by the RRT, or escalation of care to the ICU, intermediate care area or high dependency ward directly from the RRT activation. These interventions include endotracheal intubation, initiation of non-invasive ventilation, fluid resuscitation and/or initiation of vasopressors, point-of-care ultrasound and discussion of limits of treatment or resuscitation orders by the RRT. Patients were followed up until hospital discharge for the primary outcome of inpatient mortality. Patients who were discharged alive from hospital but demised within 30 days of RRT activation were also included in the primary outcome. These outcomes were referenced from our electronic medical records, which included deaths occurring in the community. The study protocol (with a waiver of consent from participants) was submitted to our hospital's institutional review board (CIRB 2018/2190).

\section{Statistical analysis}

Continuous variables were expressed as mean and standard deviation or as median and interquartile range (IQR) for non-normally distributed variables. Categorical variables were expressed as frequency and percentage. Baseline characteristics, clinical severity scores, RRT interventions and outcomes of both survivors and non-survivors were compared using independent samples Student's t-test and MannWhitney U test for continuous variables, and chi-square or Fisher's Exact test for categorical variables. Univariate and multivariate logistic regression analyses were performed to identify factors associated with in-hospital mortality. Variables associated with mortality $(P<0.20)$ were identified and analysed using stepwise backward (likelihood ratio) logistic regression analysis, keeping variables with $P<0.10$. Multicollinearity testing for variables included in the multivariable analysis was performed with variance inflation factor analysis. Results were presented as odds ratios and 95\% confidence intervals. Cut-offs for continuous variables (NEWS, CCI and ECOG performance status) that were used for the logistic regression analysis were determined based on recognised clinical significance, e.g. a NEWS score of 7 or more was high risk. All statistical analyses were performed using SPSS Statistics software version 22.0 (IBM Corp, Armonk, US).

\section{RESULTS}

\section{Patient baseline characteristics}

A total of 951 consecutive RRT activations from 869 patients were included in this study (Table 1). Preexisting comorbidities of patients for moderate to severe chronic kidney disease and malignancy were $27.6 \%$ and $38.0 \%$, respectively. A significant proportion of patients were admitted under the medical oncology $(18.3 \%)$ and haematology (12.5\%) departments, with the majority of patients admitted under internal medicine department $(43.0 \%)$. Twenty-two $(2.3 \%)$ patients had prior haematopoietic stem cell transplant. Eleven $(1.2 \%)$ and $4(0.4 \%)$ patients had prior kidney and liver transplants, respectively.

\section{Interventions and outcomes of RRT activation}

Table 2 summarises the characteristics and outcomes of RRT activations. Eighty-two (8.6\%) of RRT activations were repeat activations. The proportion of patients with medium and high-risk NEWS scores at the time of RRT activation were $30.2 \%(285 / 944)$ and $45.8 \%$ (432/944), respectively. The majority (71.8\%) of patients were referred for respiratory failure or cardiovascularrelated concerns (haemodynamic instability, tachycardia, bradycardia and arrhythmia).

At the time of RRT activation, $11.9 \%$ of patients had 1 or more limits of medical therapy established. The majority of patients $(79.8 \%)$ received 1 or more interventions by the RRT, including endotracheal intubation $(12.7 \%)$ and point-of-care ultrasound for diagnostics (17.0\%). Discussion of resuscitation status and goals of care were initiated by the RRT in 140 $(14.7 \%)$ patients, of whom $110(11.2 \%)$ had limits of medical therapy changed post-RRT activation. Approximately a quarter $(24.2 \%)$ of patients required ICU admission directly from an RRT activation. The in-hospital or 30-day mortality rate for all RRT activations was $38.1 \%$ and the median length of stay was 20 (IQR 10-41) days. Among the 869 patients who required at least 1 RRT activation during hospitalisation, the in-hospital or 30-day mortality rate was $36.6 \%$. 
Table 1. Baseline patient characteristics

\begin{tabular}{|c|c|}
\hline \multicolumn{2}{|l|}{ Characteristics $(\mathrm{N}=\mathbf{8 6 9})$} \\
\hline Age, mean (SD), years & $64(15)$ \\
\hline Male sex, no. (\%) & $469(54.0)$ \\
\hline \multicolumn{2}{|l|}{ Ethnicity, no. (\%) } \\
\hline Chinese & $620(71.3)$ \\
\hline Malay & $120(13.8)$ \\
\hline Indian & $77(8.9)$ \\
\hline Others & $52(6.0)$ \\
\hline \multicolumn{2}{|l|}{ ECOG performance status, no. (\%) } \\
\hline 0 & $236(27.2)$ \\
\hline 1 & $380(43.7)$ \\
\hline 2 & $144(16.6)$ \\
\hline 3 & $81(9.3)$ \\
\hline 4 & $28(3.2)$ \\
\hline Charlson Comorbidity Index, median (IQR) & $6(4-7)$ \\
\hline \multicolumn{2}{|l|}{ Comorbidity, no. (\%) } \\
\hline Diabetes mellitus & $310(35.7)$ \\
\hline Diabetes mellitus with end-organ damage & $128(14.7)$ \\
\hline Moderate to severe chronic kidney disease & $240(27.6)$ \\
\hline Congestive heart failure & $159(18.3)$ \\
\hline Myocardial infarction & $157(18.1)$ \\
\hline Chronic pulmonary disease & $73(8.4)$ \\
\hline Liver cirrhosis or chronic hepatitis & $117(12.3)$ \\
\hline Malignancy & $330(38.0)$ \\
\hline $\begin{array}{l}\text { Any leukaemia, lymphoma, or localised } \\
\text { solid tumour }\end{array}$ & $220(25.3)$ \\
\hline Metastatic solid tumour & $110(12.7)$ \\
\hline
\end{tabular}

ECOG: Eastern Cooperative Oncology Group

\section{Factors associated with mortality}

A comparison of clinical and RRT characteristics between survivors and non-survivors is summarised in Table 3. Non-survivors had worse clinical severity scores (CCI, ECOG and NEWS). A higher proportion of non-survivors had pre-existing metastatic or haematological cancers and were admitted to the ICU after RRT activation. A longer duration of hospitalisation at the time of RRT activation was also associated with mortality. In multivariate analysis, the above factors remained associated with mortality (Table 4, Fig. 1). On testing for multicollinearity, the average variance inflation factor of all 10 variables included in the multivariate analysis was 1.07 , indicating that no significant collinearity existed.

\section{DISCUSSION}

In this prospective observational study, the interventions and outcomes of patients who required second-tier RRT activations were investigated. A significant proportion of patients received 1 or more emergent interventions in the general ward. Approximately 1 in 4 patients were admitted to the ICU from an RRT activation, and 1 in 3 patients did not survive to hospital discharge. Older age, poorer functional status, known malignancy, higher NEWS scores, ICU admission and longer duration 
Table 2. Characteristics and outcomes of rapid response team activations

\begin{tabular}{|c|c|}
\hline \multicolumn{2}{|l|}{ RRT activation $(\mathrm{N}=951)$} \\
\hline Duration of hospitalisation at time of RRT activation, median (IQR), days & $4(1-14)$ \\
\hline Repeat RRT activation, no. (\%) & $82(8.6)$ \\
\hline RRT response time, median (IQR), minutes & $12(8-17)$ \\
\hline NEWS at time of activation, median (IQR) & $6(4-8)^{a}$ \\
\hline Low risk NEWS $(0-4)$ & $227(24.0)$ \\
\hline Medium risk NEWS (5-6 or 3 in any 1 parameter) & $285(30.2)$ \\
\hline High risk NEWS $(\geq 7)$ & $432(45.8)$ \\
\hline \multicolumn{2}{|l|}{ Reason for RRT activation, no. (\%) } \\
\hline Airway concern & $81(8.5)$ \\
\hline Respiratory failure or distress & $381(40.1)$ \\
\hline Haemodynamic instability, tachycardia/bradycardia/arrhythmias & $301(31.7)$ \\
\hline Primary team concerns or request for closer monitoring & $131(13.7)$ \\
\hline Metabolic derangements & $46(4.8)$ \\
\hline Others & $11(1.2)$ \\
\hline \multicolumn{2}{|l|}{ Limits of medical therapy (pre-RRT activation), no. (\%) } \\
\hline Full care or care limits not yet established & $838(88.1)$ \\
\hline Do not resuscitate, limit care to vasopressor support and/or dialysis & $88(9.3)$ \\
\hline Do not resuscitate, general ward management & $25(2.6)$ \\
\hline One or more interventions performed by RRT, no. $(\%)$ & $759(79.8)$ \\
\hline Endotracheal intubation & $121(12.7)$ \\
\hline Non-invasive ventilation & $44(4.6)$ \\
\hline Fluid resuscitation or vasopressor support & $146(15.4)$ \\
\hline Point-of-care ultrasound & $162(17.0)$ \\
\hline Central venous or arterial line insertion & $9(0.9)$ \\
\hline Discussion of limits of medical therapy status & $140(14.7)$ \\
\hline Limitation of medical therapy established & $99(10.4)$ \\
\hline RRT disposition: ICU & $230(24.2)$ \\
\hline RRT disposition: HD or ICA admission & $325(34.2)$ \\
\hline Length of stay, median (IQR), days & $20(10-41)$ \\
\hline 24-hour mortality, no. (\%) & $45(4.7)$ \\
\hline In-hospital mortality, no. (\%) & $332(34.9)$ \\
\hline In-hospital or 30-day mortality, no. (\%) & $362(38.1)$ \\
\hline
\end{tabular}

HD: high dependency; ICA: intermediate care area; ICU: intensive care unit; NEWS: National Early Warning Score; RRT: rapid response team

${ }^{a}$ Data missing for 7 activations 
of hospitalisation at time of RRT activation were all independently associated with mortality.

Patients requiring second-tier RRT activations appeared to be of high acuity, with many requiring specialised interventions. A significant proportion of patients had a NEWS $\geq 7$ at the time of RRT activation, and most activations were for respiratory failure and/ or haemodynamic instability or tachy-bradyarrhythmia. In addition, most patients required 1 or more emergent bedside interventions, including endotracheal intubation and escalation of care to ICU. With the large heterogeneity in patient and RRS characteristics, the outcomes of patients requiring RRT activations are currently not well established. While systematic reviews of non-tiered RRT activations reported an overall ICU admission and in-hospital mortality of approximately $23 \%$ and $26 \%$, respectively, mortality rates from individual studies ranged widely from 12 to $60 \% .^{19}$ Reported in-hospital mortality rates $(30-34 \%)$ from large single-centre studies designed to evaluate outcomes of patients requiring RRT activations were similar to our mortality outcomes..$^{20,21}$ Notably, our

Table 3. Comparison of clinical and RRT characteristics between survivors and non-survivors (in-hospital or 30-day mortality)

\begin{tabular}{|c|c|c|c|}
\hline & $\begin{array}{c}\text { Survivors } \\
(n=589)\end{array}$ & $\begin{array}{c}\text { Non-survivors } \\
(n=362)\end{array}$ & $P$ value \\
\hline Age, mean (SD), years & $64(15)$ & $65(14)$ & 0.134 \\
\hline Male sex, no. $(\%)$ & $308(52.3)$ & $210(58.0)$ & 0.086 \\
\hline ECOG performance status, median (IQR) & $1(0-1)$ & $1(1-2)$ & $<0.001$ \\
\hline \multicolumn{4}{|l|}{ Comorbidity, no. (\%) } \\
\hline Metastatic cancer or haematological cancer & $131(22.2)$ & $134(37.0)$ & $<0.001$ \\
\hline Moderate to severe chronic kidney disease & $151(25.6)$ & $113(31.2)$ & 0.062 \\
\hline Congestive heart failure & $108(18.3)$ & $70(19.2)$ & 0.701 \\
\hline Liver cirrhosis or chronic hepatitis & $58(9.8)$ & $59(16.3)$ & 0.003 \\
\hline Chronic pulmonary disease & $45(7.6)$ & $33(9.1)$ & 0.421 \\
\hline Duration of hospitalisation at time of RRT activation, median (IQR), days & $2(1-8)$ & $10(3-21)$ & $<0.001$ \\
\hline Repeat RRT activation, no. (\%) & $38(6.5)$ & $44(12.2)$ & 0.002 \\
\hline NEWS at time of activation, median (IQR) & $6(3-8)^{\mathrm{a}}$ & $7(5-9)^{b}$ & $<0.001$ \\
\hline High risk NEWS ( $\geq 7$ ) & $235(40.2)$ & $197(54.7)$ & $<0.001$ \\
\hline \multicolumn{4}{|l|}{ Reason for RRT activation, no. (\%) } \\
\hline Airway concern & $50(8.5)$ & $31(8.6)$ & 0.968 \\
\hline Respiratory failure or distress & $220(37.4)$ & $161(44.5)$ & 0.029 \\
\hline \multicolumn{4}{|l|}{ Intervention performed by RRT, no. (\%) } \\
\hline Endotracheal intubation & $57(9.7)$ & $64(17.7)$ & $<0.001$ \\
\hline Non-invasive ventilation & $28(4.8)$ & $16(4.4)$ & 0.812 \\
\hline Fluid resuscitation and/or vasopressor support & $81(13.8)$ & $65(18.0)$ & 0.083 \\
\hline RRT disposition: ICU, no. (\%) & $116(19.7)$ & $114(31.5)$ & $<0.001$ \\
\hline Hospital length of stay, median (IQR), days & $18(10-40)$ & $22(11-45)$ & 0.067 \\
\hline
\end{tabular}

ECOG: Eastern Cooperative Oncology Group; ICU: intensive care unit; NEWS: National Early Warning Score; RRT: rapid response team

${ }^{\text {a }}$ Data missing for 5 activations

${ }^{\mathrm{b}}$ Data missing for 2 activations 
Table 4. Univariate and multivariate analysis of factors associated with in-hospital or 30-day mortality

\begin{tabular}{|c|c|c|c|c|}
\hline & Odds ratio $(95 \% \mathrm{CI})$ & $P$ value & Odds ratio $(95 \% \mathrm{CI})$ & $P$ value \\
\hline Age $\geq 65$ years & $1.28(0.98-1.66)$ & 0.072 & $1.51(1.12-2.04)$ & 0.006 \\
\hline Male sex & $1.26(0.97-1.64)$ & 0.086 & - & - \\
\hline ECOG performance status $\geq 3$ & $2.45(1.65-3.63)$ & $<0.001$ & $2.24(1.45-3.46)$ & $<0.001$ \\
\hline Metastatic cancer & $1.37(0.93-2.01)$ & 0.112 & $2.64(1.71-4.08)$ & $<0.001$ \\
\hline Haematological cancer & $2.33(1.63-3.33)$ & $<0.001$ & $2.78(1.84-4.19)$ & $<0.001$ \\
\hline Myocardial infarction & $1.34(0.96-1.86)$ & 0.086 & $1.45(1.00-2.09)$ & 0.050 \\
\hline Moderate to severe chronic kidney disease & $1.15(0.99-1.33)$ & 0.062 & $1.23(1.04-1.45)$ & 0.014 \\
\hline Liver cirrhosis or chronic hepatitis & $1.78(1.21-2.63)$ & 0.004 & $1.95(1.27-3.00)$ & 0.002 \\
\hline Charlson Comorbidity Index $\geq 6$ & $1.78(1.37-2.32)$ & $<0.001$ & - & - \\
\hline Duration of hospitalisation, per 10-day increase & $1.26(1.16-1.37)$ & $<0.001$ & $1.26(1.16-1.37)$ & $<0.001$ \\
\hline NEWS $\geq 7$ & $1.80(1.38-2.34)$ & $<0.001$ & $1.55(1.16-2.08)$ & $<0.001$ \\
\hline Referral for respiratory failure or distress & $1.34(1.03-1.75)$ & 0.030 & - & - \\
\hline Intubation performed by RRT & $2.00(1.37-2.94)$ & $<0.001$ & - & - \\
\hline Fluid resuscitation or initiation of vasopressor support by RRT & $1.37(0.96-2.96)$ & 0.084 & - & - \\
\hline RRT disposition: ICU & $1.87(1.39-2.53)$ & $<0.001$ & $1.82(1.31-2.55)$ & $<0.001$ \\
\hline
\end{tabular}

CI: confidence interval; ECOG: Eastern Cooperative Oncology Group; ICU: intensive care unit; NEWS: National Early Warning Score; RRT: rapid response team

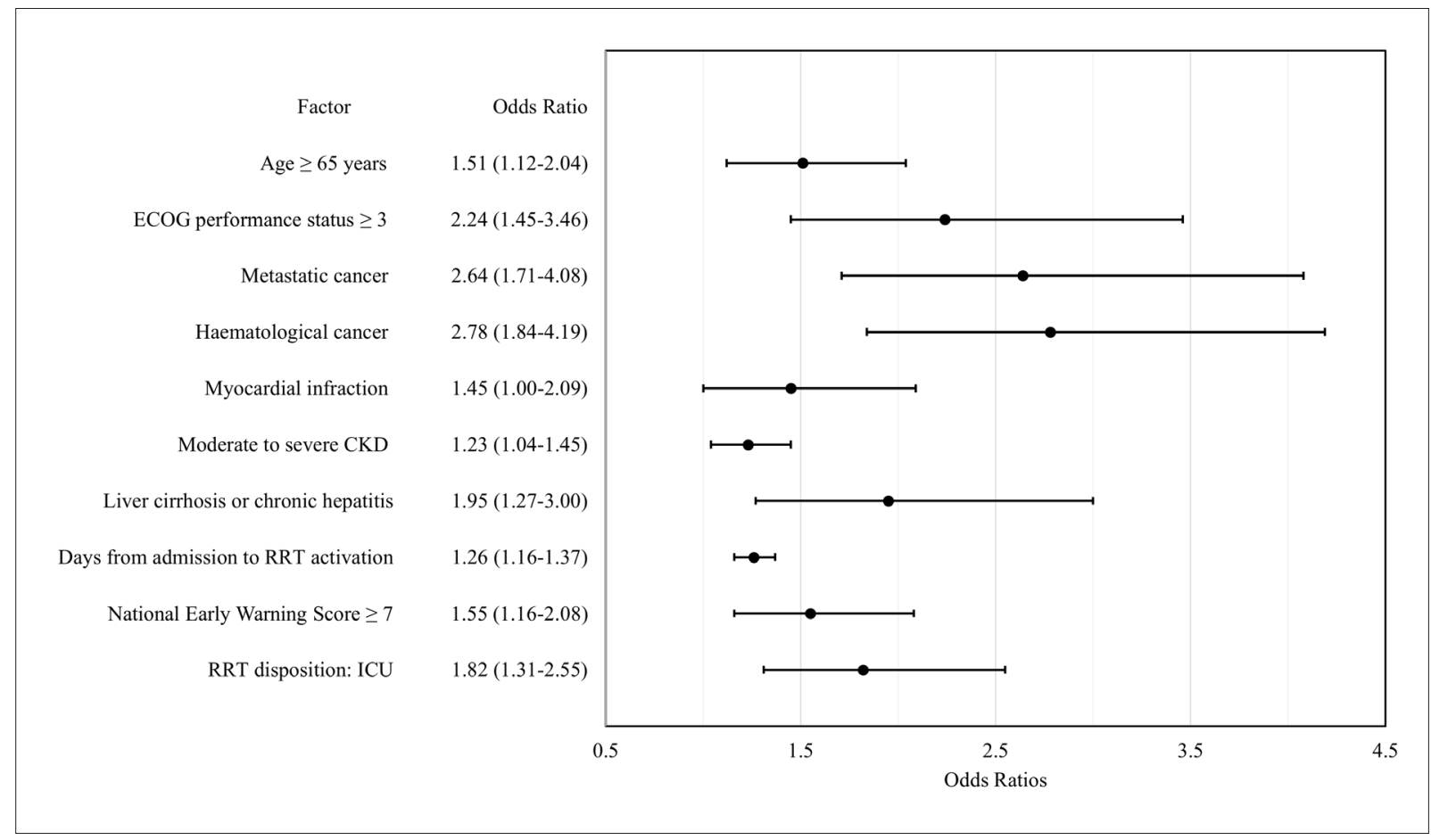

Fig. 1. Multivariate analysis of factors associated with in-hospital or 30-day mortality.

CKD: chronic kidney disease; ECOG: Eastern Cooperative Oncology Group; ICU: intensive care unit; RRT: rapid response team 
centre is a tertiary hospital with a national cancer centre. Nearly half of our patients had underlying cancer, which was likely to have contributed to the significant mortality rates observed. Nevertheless, our study highlighted the high acuity of patients who required second-tier RRT responses, reinforcing the need for specialised and dedicated (without competing clinical responsibilities) RRT members, who are a cornerstone of efficient RRS to maximise delivery of timely and effective interventions.

Specialised services, however, increase the strain on limited critical care resources. The premise behind a second-tier response therefore is that first-tier responses by a ward team is effective in identifying and correcting early states of deterioration, or establishing goals of care to reduce unnecessary escalation, thereby reserving specialised RRT services to acutely deteriorating patients. Early involvement of the ward team may also address concerns with autonomy, de-skilling and continuity of care. Hospitals implementing a tiered response have reported more efficient use of resources, and even improved patient outcomes. ${ }^{10,22}$ The main concern with a tiered response, however, are delayed activations and interventions, which have been shown to be associated with worse clinical outcomes. ${ }^{23}$ Furthermore, a high-risk NEWS $\geq 7$ at the time of RRT activation was associated with increased mortality in our patient cohort. This reinforces the emphasis on early activation, especially in hospitals implementing second-tier RRTs. Considering the high acuity and mortality of patients with second-tier RRT activations, it will be important to identify cases with delayed first-tier or subsequent second-tier activations, and address potential barriers to activation. The implementation of early warning scores like NEWS, which has been validated in a Singapore hospital to predict critical illness in patients admitted to an acute medical ward, ${ }^{24}$ may help to reduce delayed activations. These early warning scores serve as the afferent limb of the RRS. Unfortunately, evaluating the effectiveness of a RRS or RRT is challenging due to the inherent complexity and heterogeneity of strategies implemented and interactions within each healthcare institution. ${ }^{6,25}$ Indices such as "healthy" RRT utilisation rates have therefore been proposed as surrogates for a mature and effective RRS. ${ }^{26}$ Ultimately, monitoring patient outcomes, RRT utilisation, process indicators such as timely activation, and review of cases with delayed activation will be important to ensure that an RRS remains timely and effective.

Predicting poor outcomes at the time of activation is also important for RRTs to facilitate timely transfers to ICUs, optimise triage decisions and guide discussions on goals of care. Older age, deranged vital signs and longer duration of hospitalisation at RRT activation were found to be associated with in-hospital mortality in a retrospective analysis of large registry data by Shappell et al. ${ }^{27}$ These were consistent with our study findings. In addition, metastatic cancer and haematological cancer as comorbidities were significantly associated with mortality, independent of age, performance status and physiological deterioration based on NEWS. In patients with cancer, reported in-hospital mortality after RRT activations were $33-40 \%,{ }^{28-30}$ with mortality rates as high as $42 \%$ demonstrated in patients with haematological cancer. ${ }^{31}$ Clearly, patients with cancer were at a high risk of dying, especially so if RRT activations were required. ${ }^{32,33}$ However, patients with cancer were widely heterogenous, with differences in cancer type, cancer staging, treatment options and response to cancer therapy. As cancer patients are living longer with access to improved cancer treatment, further studies are needed to evaluate how the above factors influence patient outcomes. This will help empower RRTs to make evidence-based triage decisions and guide discussions on goals of care.

There is increasing interest in leveraging RRTs to improve end-of-life care and avoid futile interventions, ${ }^{34-36}$ with some studies reporting improved end-of-life care such as reduced pain and patient distress. ${ }^{37,38}$ Considering the high patient acuity and mortality outcomes, discussions on resuscitation status and goals of care, when appropriate, are an important function of a second-tier response RRT. In our cohort, RRT-initiated discussion on resuscitation status resulted in a change in limits of medical therapy in a majority of cases. Notably, poor ECOG performance was found to be significantly associated with increased mortality. Similar associations have been reported when assessing patient frailty (by now a well-recognised clinical entity associated with mortality), increased healthcare utilisation and disability. ${ }^{32,39,40}$ As such, poor prognostic markers such as poor functional status, frailty or comorbidities such as metastatic cancer should guide discussions on goals of care, or trigger palliative services to improve end-oflife care.

There were limitations to our study. As this was a single-centre study at a tertiary hospital providing specialised care such as solid organ, haematological transplants and cancer therapy, generalisability of the results is limited. Only RRT activations from patients admitted under medical disciplines were included. Nighttime RRT activations, which appear to be 
associated with higher risks of mortality, ${ }^{21,41}$ were not included. The absence of aggregate risk scores like NEWS in our hospital raises the question of whether delayed first-tier responses may have contributed to poorer outcomes. At the time of activation, NEWS was documented by the RRT but not used routinely in all general ward patients as part of the RRS afferent limb in our institution. Plans are currently being made to implement an early warning system. Finally, our study did not include information on first-tier activations, such as its number, patient characteristics and time between first- and second-tier activations, to identify delayed responses and associated outcomes. In our institution, ward teams were encouraged to activate the RRT if they were concerned or escalation of care was deemed likely, even in the absence of significant physiological deterioration.

\section{CONCLUSION}

To the best of our knowledge, this is the first study describing RRT intervention and patient outcomes in Singapore. Escalation of care and emergent interventions are common with second-tier RRTs. We highlight the need for second-tier RRTs to have dedicated and specialised critical care capability and measures to avoid delayed activations, including the use of early warning scores as the afferent limb of the RRS. In patients requiring RRT activations, prognostic markers such as poor functional performance status and metastatic or haematological cancer are helpful in guiding triage decisions and goals of care discussion.

\section{Acknowledgements}

We thank all nursing staff, respiratory therapists, and healthcare workers from the Medical Intensive Care Unit and Department of Respiratory and Critical Care Medicine at Singapore General Hospital, without whom our rapid response service would not have been possible.

\section{REFERENCES}

1. Mitchell OJL, Motschwiller CW, Horowitz JM, et al. Rapid response and cardiac arrest teams: A descriptive analysis of 103 American hospitals. Crit Care Explor 2019;1:e0031.

2. Dukes K, Bunch JL, Chan PS, et al. Assessment of rapid response teams at top-performing hospitals for in-hospital cardiac arrest. JAMA Intern Med 2019;179:1398-405.

3. Lyons PG, Edelson DP, Churpek MM. Rapid response systems. Resuscitation 2018;128:191-7.

4. Maharaj R, Raffaele I, Wendon J. Rapid response systems: a systematic review and meta-analysis. Crit Care 2015;19:254.

5. Solomon RS, Corwin GS, Barclay DC, et al. Effectiveness of rapid response teams on rates of in-hospital cardiopulmonary arrest and mortality: A systematic review and meta-analysis. J Hosp Med 2016;11:438-45.

6. Salvatierra GG, Bindler RC, Daratha KB. Rapid response teams: is it time to reframe the questions of rapid response team measurement? J Nurs Scholarsh 2016;48:616-23.

7. Hillman K, Chen J, Cretikos M. Introduction of the medical emergency team (MET) system: a cluster-randomised controlled trial. Lancet 2005;365:2091-7.

8. Karpman C, Keegan MT, Jensen JB, et al. The impact of rapid response team on outcome of patients transferred from the ward to the ICU: a single-center study. Crit Care Med 2013;41:2284-91.

9. Ko BS, Lim TH, Oh J, et al. The effectiveness of a focused rapid response team on reducing the incidence of cardiac arrest in the general ward. Medicine 2020;99:e19032.

10. Study Investigators TCMETM; Concord Medical Emergency Team (MET) 2 Study Investigators. Outcomes following changing from a two-tiered to a three-tiered hospital rapid response system. Aust Health Rev 2019;43:178-87.

11. Aitken LM, Chaboyer W, Vaux A, et al. Effect of a 2-tier rapid response system on patient outcome and staff satisfaction. Aust Crit Care 2015;28:107-15.

12. Aneman A, Frost SA, Parr MJ, et al. Characteristics and outcomes of patients admitted to ICU following activation of the medical emergency team: impact of introducing a two-tier response system. Crit Care Med 2015;43:763-73.

13. Bevan C, Officer C, Crameri J, et al. Reducing "cry wolf" - changing trauma team activation at a pediatric trauma centre. J Trauma 2009;66:698-702.

14. Jenkins P, Rogers J, Kehoe A, et al. An evaluation of the use of a two-tiered trauma team activation system in a UK major trauma centre. Emerg Med J 2015;32:364-7.

15. Rehn M, Lossius HM, Tjosevik KE, et al. Efficacy of a two-tiered trauma team activation protocol in a Norwegian trauma centre. Br J Surg 2012;99:199-208.

16. Smith GB, Prytherch DR, Meredith P, et al. The ability of the National Early Warning Score (NEWS) to discriminate patients at risk of early cardiac arrest, unanticipated intensive care unit admission, and death. Resuscitation 2013;84:465-70.

17. Charlson ME, Pompei P, Ales KL, et al. A new method of classifying prognostic comorbidity in longitudinal studies: development and validation. J Chronic Dis 1987;40:373-83.

18. Oken MM, Creech RH, Tormey DC, et al. Toxicity and response criteria of the Eastern Cooperative Oncology Group. Am J Clin Oncol 1982;5:649-55.

19. Tirkkonen J, Tamminen T, Skrifvars MB. Outcome of adult patients attended by rapid response teams: A systematic review of the literature. Resuscitation 2017;112:43-52.

20. Fernando SM, Fox-Robichaud AE, Rochwerg B, et al. Prognostic accuracy of the Hamilton Early Warning Score (HEWS) and the National Early Warning Score 2 (NEWS2) among hospitalized patients assessed by a rapid response team. Crit Care 2019;23:60.

21. Fernando SM, Reardon PM, Bagshaw SM, et al. Impact of nighttime Rapid Response Team activation on outcomes of hospitalized patients with acute deterioration. Crit Care 2018;22:67.

22. Howell MD, Ngo L, Folcarelli R, et al. Sustained effectiveness of a primary team based rapid response system. Crit Care Med 2012;40:2562-68. 
23. Gupta S, Green C, Subramaniam A, et al. The impact of a delayed rapid response call activation on patient outcomes. J Crit Care 2017;41:86-90.

24. Lim WT, Fang AH, Loo CM, et al. Use of the National Early Warning Score (NEWS) to identify acutely deteriorating patients with sepsis in acute medical ward. Ann Acad Med Singap 2019;48:145-49.

25. Olsen SL, Søreide E, Hillman K, et al. Succeeding with rapid response systems - a never ending process: A systematic review of how health-care professionals perceive facilitators and barriers within the limbs of the RRS. Resuscitation 2019;144:75-90.

26. Jones D, Bellomo R, DeVita MA. Effectiveness of the medical emergency team: the importance of dose. Crit Care 2009;13:313.

27. Shappell C, Snyder A, Edelson DP, et al. Predictors of in-hospital mortality after rapid response team calls in a 274 hospital nationwide sample. Crit Care Med 2018;46:1041-8.

28. Lee J, Ban WH, Kim SW, et al. Utilization of a rapid response team and associated outcomes in patients with malignancy. Acute Crit Care 2020;35:16-23.

29. Austin CA, Hanzaker C, Stafford R, et al. Utilization of rapid response resources and outcomes in a comprehensive cancer center. Crit Care Med 2014;42:905-9.

30. Laothamatas KC, Bekker TD, Leiby BE, et al. Mortality outcomes in hospitalized oncology patients after rapid response team activation. JCSO 2018;16:e250-5.

31. Gershkovich B, Fernando SM, Herritt B, et al. Outcomes of hospitalized hematologic oncology patients receiving rapid response system activation for acute deterioration. Crit Care 2019;23:286.

32. Cardona-Morrell M, Chapman A, Turner RM, et al. Pre-existing risk factors for in-hospital death among older patients could be used to initiate end-of-life discussions rather than rapid response system calls: A case-control study. Resuscitation 2016;109:76-80.

33. White K, Bernard A, Scott I. Derivation and validation of a risk score for predicting mortality among inpatients following rapid response team activation. Postgrad Med J 2019;95:300-6.

34. Jäderling G, Bell M, Martling CR, et al. Limitations of medical treatment among patients attended by the rapid response team. Acta Anaesthesiol Scand 2013;57:1268-74.

35. Jones D, Moran J, Winters B, et al. The rapid response system and end-of-life care. Curr Opin Crit Care 2013;19:616-23.

36. Coombs MA, Nelson K, Psirides AJ, et al. Characteristics and dying trajectories of adult hospital patients from acute care wards who die following review by the rapid response team. Anaesth Intensive Care 2016;44:262-9.

37. Vazquez R, Gheorghe C, Gregoriyan A, et al. Enhanced end-of-life care associated with deploying a rapid response team: a pilot study. J Hosp Med 2009;4:449-52.

38. Downar J, Barua R, Rodin D, et al. Changes in end of life care 5 years after the introduction of a rapid response team: a multicentre retrospective study. Resuscitation 2013;84:1339-44.

39. So RKL, Bannard-Smith J, Subbe CP, et al. The association of clinical frailty with outcomes of patients reviewed by rapid response teams: an international prospective observational cohort study. Crit Care 2018;22:227.

40. De Biasio JC, Mittel AM, Mueller AL, et al. Frailty in critical care medicine: a review. Anesth Analg 2020;130:1462-73.

41. Churpek MM, Edelson DP, Lee JY, et al. Association between survival and time of day for rapid response team calls in a national registry. Crit Care Med 2017;45:1677-82. 